\title{
La imagen como memoria: Palestina patrimonio invisible
}

\author{
The image as a memory: Palestine invisible heritage
}

\author{
Enviado em: 13/05/2018 \\ Aceito em: 27/07/2018 \\ Jenny González-Muñoz ${ }^{1}$
}

\begin{abstract}
Resumen:
El presente ensayo visual pretende mostrar la importancia de la ilustración como elemento vinculado a la memoria social, a partir de la re-lectura de sus creadoras y creadores en relación a temas que involucran agresiones a los pueblos históricamente perseguidos, por razones religiosas o político-territoriales, tal el caso del palestino. En esta muestra nuestro abordaje se concentra en el patrimonio cultural inmaterial y su vulnerabilidad en casos de conflicto, y cómo es abordado desde la imagen como soporte de memoria.
\end{abstract}

Palabras clave: Identidad; refugiados, patrimonio cultural inmaterial.

\begin{abstract}
:
The present visual essay aims to show the importance of illustration as an element linked to social memory, based on the re-reading of its creators and creators in relation to issues that involve aggressions against historically persecuted peoples, for religious or political reasons. territorial, such as the case of the Palestinian. In this sample, our approach focuses on the intangible cultural heritage and its vulnerability in cases of conflict, and how it is approached from the image as memory support.
\end{abstract}

Keywords: Identity; refugees, intangible cultural heritage.

En una experiencia realizada en Venezuela entre 2014 y 2016 por parte de mujeres artistas en solidaridad con causas sociales mundiales, con énfasis en problemáticas relacionadas con tipos de discriminación a partir de género, religión y elementos políticoterritoriales, como el caso de Saharaui y Palestina, llevamos a cabo varias actividades en

${ }^{1}$ Post-doctoranda en Historia (PNPD-CAPES-Universidad de Passo Fundo). Doctora en Cultura y Arte para América Latina y del Caribe (UPEL-Venezuela). Magíster en Memoria Social y Patrimonio Cultural (UFPELCAPES). Licenciada en Artes (UCV-Venezuela) 
lugares no convencionales, como plazas, centro comerciales, organizaciones comunales, entre otros, en los que por medio de charlas informativas y la incorporación de poesía, música, pintura, diseño, teatro, se logró establecer un diálogo hermenéutico, con la finalidad de proporcionar herramientas para el debate desde el conocimiento de los hechos históricos, como parte de la memoria contemporánea, en un proceso de sensibilización y concienciación de las situaciones mundiales, ya no solo locales y/o regionales.

Memoria. Borrar a Palestina de la memoria de las sociedades contemporáneas. Quema de libros de Historia Palestina. Negación de Palestina como Estado. Ausencia en mapas del territorio palestino. Territorialidad. Robo de símbolos. Resistencia del pueblo palestino ante el embate israelí. La llave que abrirá la casa. El pañuelo que ampliará las fronteras. El idioma que comunicará. Procesos de identidad. Gaza. Hebrón. Nablus. Haifa. Jaffa. Qalqilya. También Jericó. Nazareth. Jerusalém. El muro del apartheid. Checkpoint. Contínua violación a los derechos humanos. Ojos ciegos de organismos internacionales. Negación de la historia. Pueblo sin tierra. Espacio vacío. 1947 legalización de la repartición. 1948 Al Nakba. Mutilación sistemática de territorios palestinos. Limpieza étnica. Discriminación. Exclusión. Ocupación. Son estas palabras provocadoras que muestran brevemente la historia de una ocupación presente en la contemporaneidad. 
Figura 1. Handala, símbolo de resistencia del pueblo palestino.

Bataker.

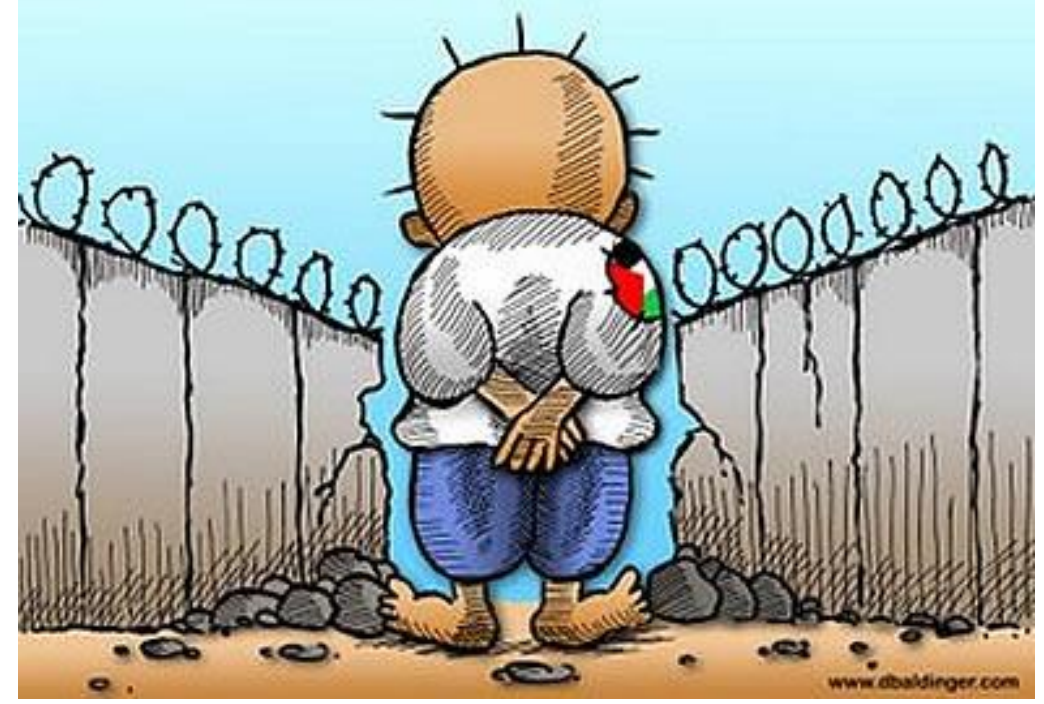

Fuente: http://www.palestinalibre.org/articulo.php?a=39680

En 1969 Nayi al Ali, refugiado palestino en el sur de Líbano, dibuja un niño descalzo que camina con las manos hacia atrás, sobre un suelo árido con algunas piedras, cercado por el muro del apartheid construido por Israel, el cual, contraviniendo leyes internacionales, ${ }^{2}$ en vez de disminuir o desaparecer, cada vez acrecienta más su extensión. Handala, es la representación del refugiado palestino (lleva un parche con la bandera en la camisa blanca que a su vez, simboliza la paz); se dice que tiene 10 años, edad que tenía su creador cuando debió salir de su tierra junto con su familia, en 1948, cuando fuera creado el Estado de Israel, por parte de la ONU, a través de la Resolución 181.

Siendo el tema de los refugiados una problemática que continua con bastante ahínco en el siglo XXI, es interesante el trabajo (inédito), en el año 2015, del joven escritor venezolano Miguel Antonio Guevara, quien realiza una entrevista a Belat Hadayed, para la fecha estudiante de la Escuela Latinoamericana de Medicina (ELAM), ${ }^{3}$ oriundo de la Franja de Gaza, y que para el momento de la conversación contaba 19 años de edad. Cuando se le pregunta, cómo era su vida en Gaza, expresa: "En Gaza estábamos rodeados de azul, el

\footnotetext{
${ }^{2}$ Se recomienda leer el artículo El Muro del Apartheid en Palestina y el Derecho Internacional, donde se detalla nombre y fecha de cada declaración y convención violada con su construcción. Disponible en: https://www.nodo50.org/csca/agenda2004/palestina/muro 18-02-04.html. Consulta en: 10 de mayo de 2018.

${ }^{3}$ Nos referimos a la Escuela Latinoamericana de Medicina Dr. Salvador Allende, ubicada en Caracas, Venezuela, en la que ingresaron estudiantes palestinos, por medio de un convenio con su gobierno.
} 
cielo, las escuelas, los hospitales, todo tenía un color azul. Desde que nací, donde volteaba veía algo azul; el hospital azul, la clínica azul, y luego me di cuenta, estando más grande: el azul es el color de las Naciones Unidas. Ahí descubrí que yo era un refugiado". ${ }^{4}$

Figura 2. Ilustración de Etten Carvallo, 2014.

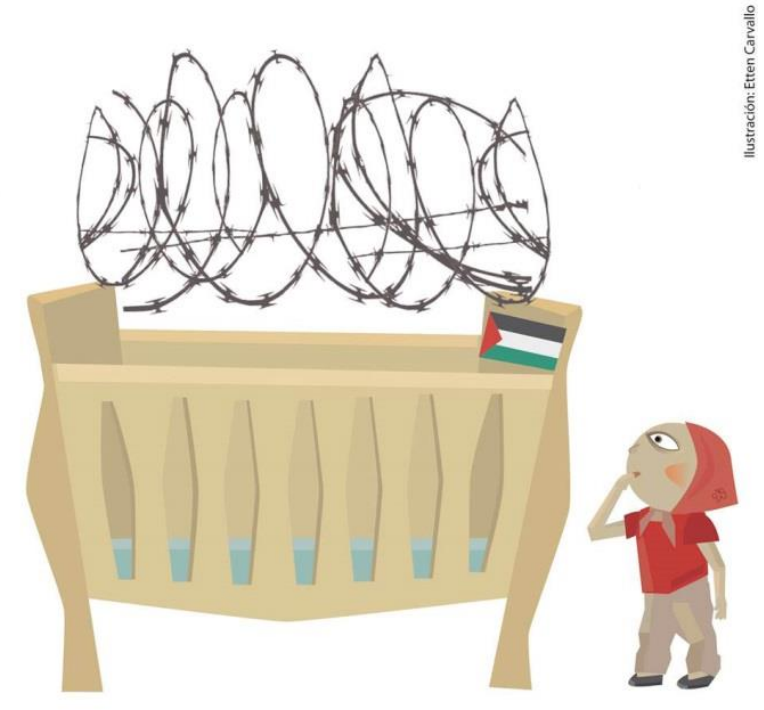

Fuente: Acervo personal de la ilustradora.

Para la artista musulmana-venezolana Etten Carvallo, la patria es simbolizada por una gran cuna de colchón azul, cercada por el alambrado presente en la vida cotidiana de palestinos, la que les impide entrar, regresar, aunque existan leyes que exigen su retorno ${ }^{5}$; desde abajo está la niña a la que no le es posible ese retorno. En la ilustración de igual modo, se resalta la religión musulmana, de modo que es plausible ver al símbolo desde su significado cognitivo, afectivo y moral; con la creencia = fe=esperanza divina; en cuanto al afectivo, todo lo que tiene que ver con la memoria, la tradición y la identidad; y en el último caso, el valor de la lucha emancipadora (GEERTZ, 2004, citado por MONTERO, 2014).

Siguiendo con la misma proporción de vulneración de niños y niñas, es interesante el trabajo mostrado en la figura siguiente, pues se destaca de nuevo la niña en peligro, en este caso, en una representación interesante que hace una analogía entre lo que debería estar haciendo y lo que la guerra le ha obligado. La niña juega a la rayuela, mientras la sombre de

${ }^{4}$ Fuente donada por el escritor a la autora de este ensayo.

${ }^{5}$ Se trata de la Resolución 194 de la ONU. 
un gran misil destruye algo más que la inocencia y los sueños de infancia. Es el futuro de una etnia, pero también de una cultura, garantizado en la procreación del vientre.

Figura 3. Niña jugando entre una ciudad que será destruida.

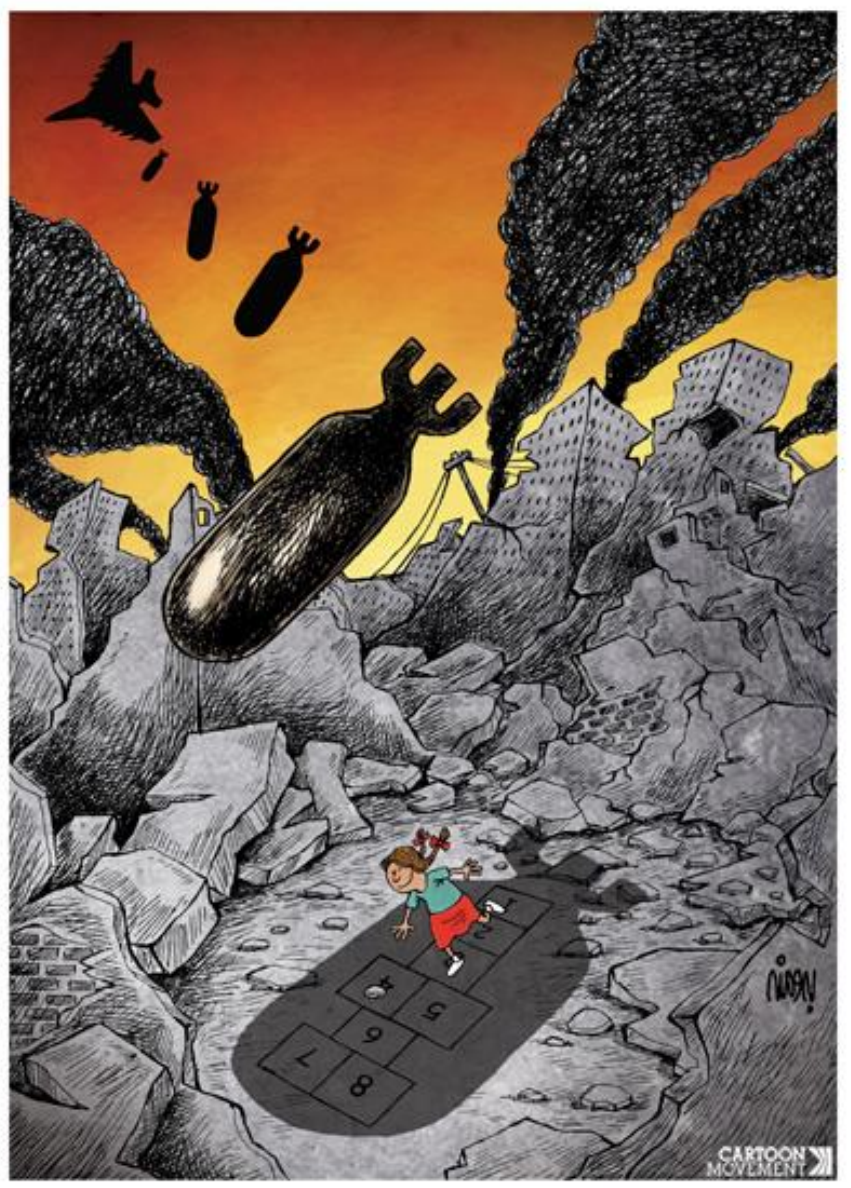

Fuente:http://fotografia.islamoriente.com/sites/default/files/image field/guerra\%20\%28Caricat ura\%29.jpeg 
Figura 4. Ilustración de Etten Carvallo, 2014.

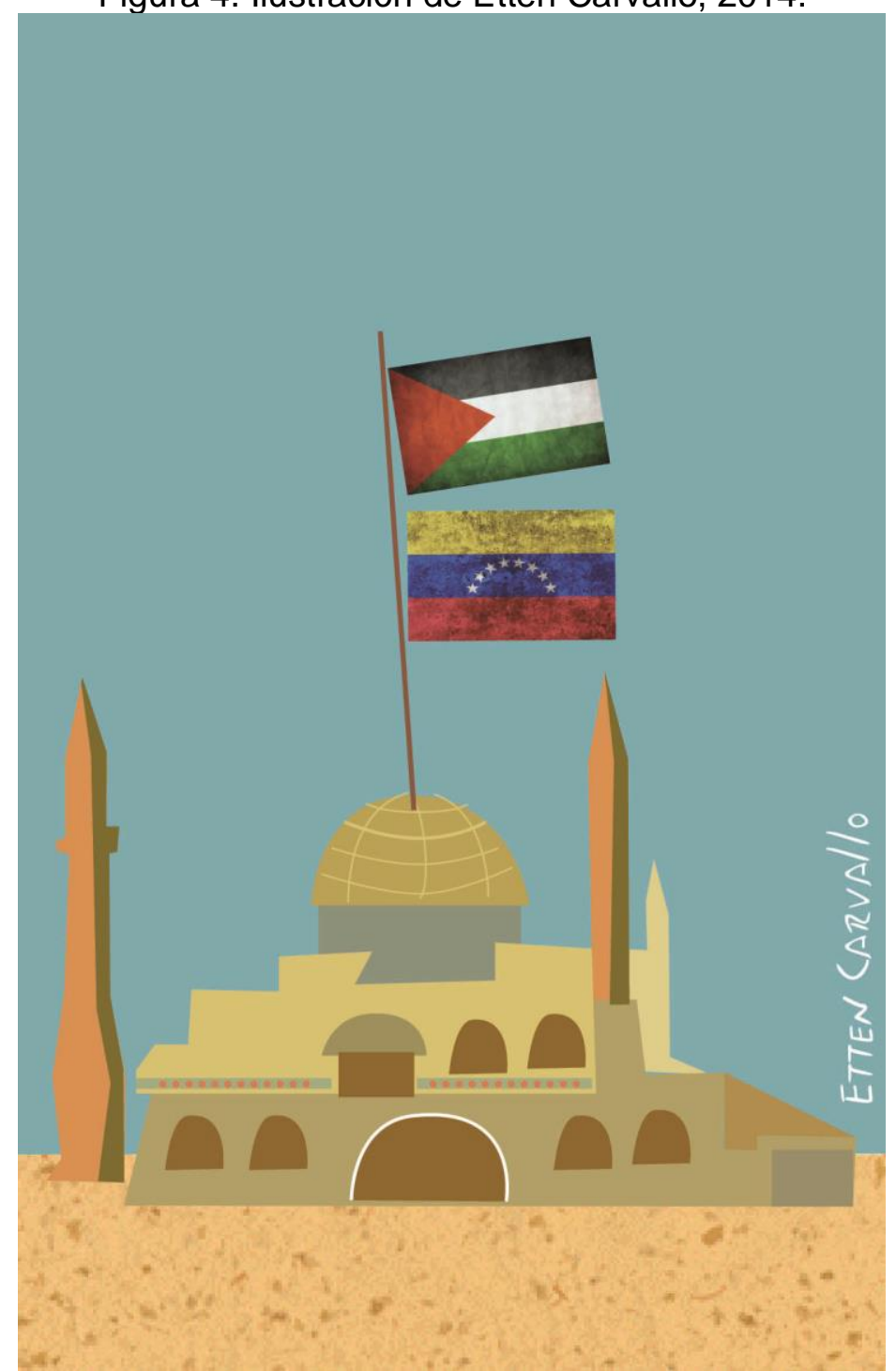

Fuente: Diario Ciudad CCS, 07/12/2017.

Cuando se habla de patrimonio cultural inmaterial, se está frente a un bien relacionado con la manifestación directamente vinculada con la persona que lo porta, como herencia, incluso, para quienes le seguirán. De modo que la vulnerabilidad de la cultura inmaterial es mayor que la material, también porque se localiza en algo referido a la creación, la memoria humana, la oralidad, imposible de restaurar, y por ello con mayor incidencia a la desaparición de su legado. En la Convención para la Salvaguardia del Patrimonio Cultural Inmaterial 2003 (UNESCO), se habla de la obligación de salvaguardar 
este dicho patrimonio, por medio, entre otras cosas, del respeto a las tradiciones, usos, costumbres, creencias; entendiendo como salvaguarda medidas, como las valorativas, por ejemplo, lo cual da paso, insoslayablemente, al resguardo de portadores de cultura. ${ }^{6}$

En la figura precedente la artista muestra un juego de imagen entre Venezuela y Palestina, aludiendo en la viñeta la relación de solidaridad entre ambos pueblos, representada en las banderas ondulando en la cúpula del Capitolio de Caracas, entre las columnas sagradas de Jerusalén, colocadas en la geografía desértica árabe, en un mismo cielo. Ambas representaciones tienen que ver con la identidad, no solo política, sino territorial y religiosa; una construcción cultural aparentemente distante, pero con características de importancia, incluso patrimonial, que pertenece al mundo global. De manera que la imagen se muestra para invitar al espectador a ir más allá de los primeros planos, describiendo un entrelineado con múltiples posibilidades de interpretación, lo cual, obviamente, se desprende desde cada aspecto cultural.

Tanto en los casos de las herencias culturales inmateriales de refugiados (culinaria, tradiciones, religiosidad, idioma, etc.), como en monumentos y otros elementos de la cultura material, ya en 1954 se establece la Convención para la Protección de los bienes culturales en caso de conflicto armado (La Haya) ${ }^{7}$, en la que se explica desde negociaciones hasta modos de salvaguardar bienes culturales de carácter patrimonial en caso, incluso, de ocupación, lo cual, no incluye a los portadores de cultura, pero da paso a la formulación de alternativas para la conservación de dicho patrimonio, que tal como asevera Mario Chagas (2009) su importancia va más allá del propio objeto, pues es espiritual..

En este sentido, tanto para la poeta venezolana Zahrah Baar, como para la ilustradora Etten Carvallo, el tema de la memoria salvaguardada como parte de un proceso cultural, es una tarea mundial interminable: "en la noche en Gaza / no pueden ocultar la matanza / no pueden ocultar el rostro asesino" (Fragmento de poema inédito de Baar, "Es conocida por todos, aquí en Gaza").

${ }^{6}$ Para ahondar en el tema: http://portal.unesco.org/es/ev.php-

URL ID=17716\&URL DO=DO TOPIC\&URL SECTION=201.html

${ }^{7}$ Para ahondar en el tema: https://www.icrc.org/spa/resources/documents/misc/treaty-1954-haque-conventioncultural-property-5tdm2q.htm 
Figura 5. Ilustración de Etten Carvallo, 2014.

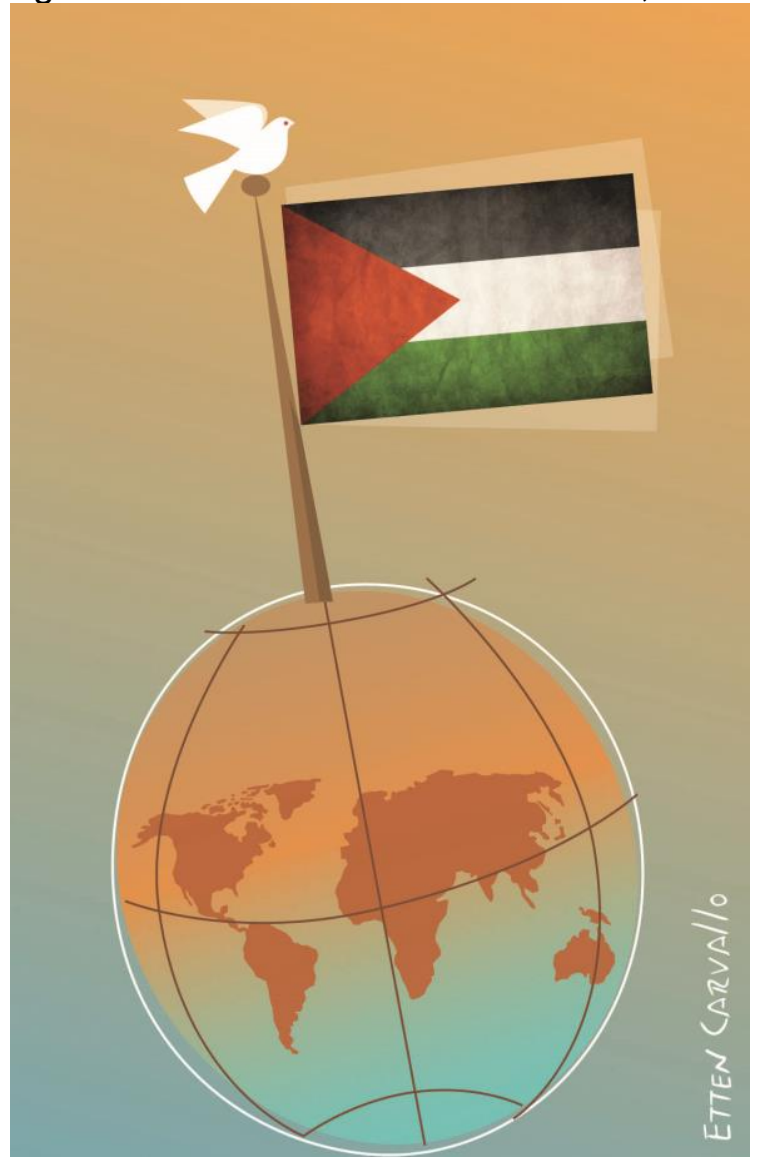

Fuente: Diario Ciudad CCS, 14/12/2017.

La globalización muestra continentes apartados y aparentemente homogéneos, no buscando una igualdad desde la horizontalidad y el respeto al "otro" (TODOROV, 2010), sino promoviendo la supremacía de poder hegemónico, buscando invisibilizar al oprimido, a pueblos minoritarios, en una historia que se repite, en cada punto geográfico con sus características y momentos, pero teniendo como leivmotiv la instauración progresiva de un borrar de la memoria social e histórica bienes de la cultura material y manifestaciones de la inmaterial, en todo caso, pueblos y sus construcciones, dando paso a nuevas estructuras de esclavización, en el caso de la contemporaneidad, otra forma de coloniaje. Aun así, el clamor debe ser, tal como en la ilustración, el ondear de la paz, sobre banderas, mapas y espacios, dando cabida a un mundo no unilateral, sino abierto realmente al respeto de la diversidad cultural en todas sus manifestaciones. 


\section{A modo de conclusión}

Figura 6 llustración de Etten Carvallo, 2013.

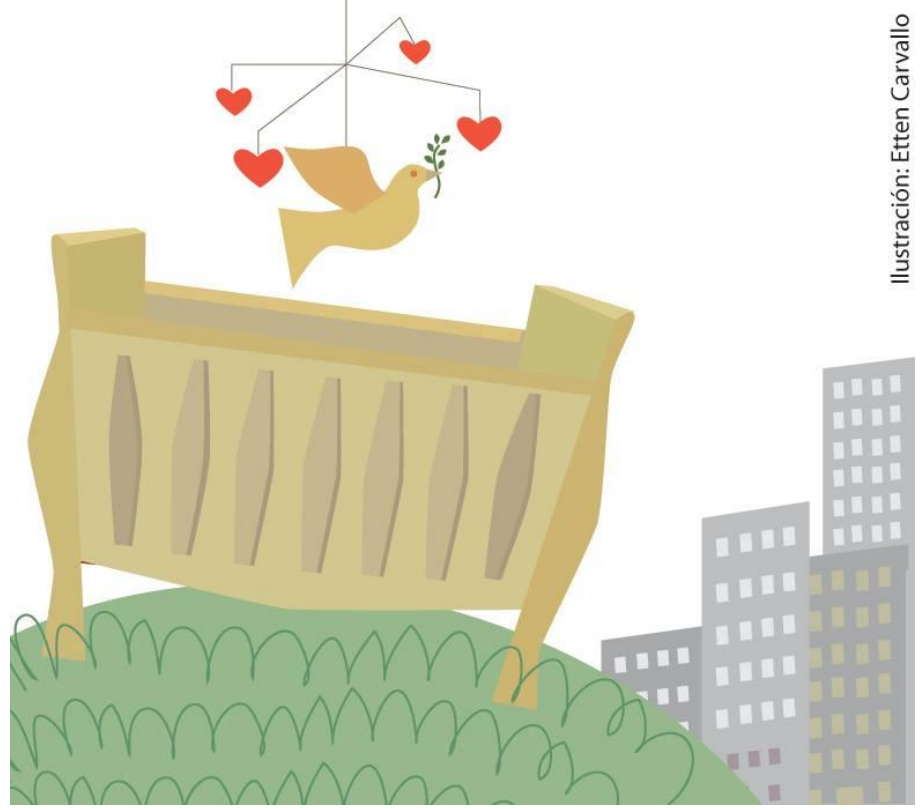

Fuente: Acervo de la artista.

¿Qué es la Patria? Tal lo expresado en la imagen, puede ser considerada como la cuna, por ser el primer objeto hecho para guardar de los peligros a quien allí descansa; es el refugio; una suerte de madre. Vemos en esta representación el paralelismo con las llamadas "raíces", es decir, el árbol que sirve para asentar al humano en un lugar que hace posible el desarrollo de varias etapas (muchas veces todas) del individuo social, es el lugar antropológico (AUGÉ, 1993) en el que se da la vida y también la muerte. Por ello, la patria se asume desde un sentimiento vinculado con el amor, en configuración con el entorno al que se "pertenece", es decir, la identidad, lo cultural; siendo que los procesos culturales de herencia inmaterial viajan con el propio humano constituyendo soportes de su memoria social, la condición de refugiados por consecuencia de conflictos de guerra u ocupación, en el caso concreto de Palestina, se afianza en una nueva construcción para salvaguardar su herencia cultural evitando así el olvido sistemático y la invisibilización de sus patrimonios.

\section{Referencias:}


AUGÉ, Marc. Los “no lugares”, espacios del anonimato. Una antropología sobre la modernidad. Barcelona: Gedisa, 1993.

CANDAU, Joël .Memória e identidade. São Paulo: Contexto, 2012.

CHAGAS, Mário. O pai Macunaíma e o Patrimônio espiritual. IN: CHAGAS, Mário; ABREU, Regina. Memória e patrimônio. Ensaios contemporâneos. $2^{\circ}$ edic. Rio de Janeiro: Lamparina, 2009.

TODOROV, Tzvetan. 0 medo dos bárbaros. Para além do choque das civilizações. Petrópolis, RJ: Editora Vozes, 2010.

MARCHI, Riccardo; BRUNO, Guido. A extrema-direita europeia perante a crise dos refugiados. Relações Internacionais, Lisboa, n. 50, p. 39-56, jun. 2016 . Disponible em: http://www.scielo.mec.pt/scielo.php?script=sci arttext\&pid=S164591992016000200004\&lng=pt\&nrm=iso Consulta en: 10 mayo 2018.

MONTERO, Paula. A teoria do simbólico de Durkheim e Lévi-Strauss: desdobramentos contemporâneos no estudo das religiões. Em: Novos estudos CEBRAP Nº 98, marzo, São Paulo, 2014. Disponibleen: https://dx.doi.org/10.1590/S0101-33002014000100007. Consulta en: 01 mayo de 2018. 